\title{
Psychoactive Substances and Drugs-Medicament and Narcotic
}

\author{
Iwona Rybakowska ${ }^{1 *}$, Bartłomiej Łukasz ${ }^{1}$, Magdalena Gregorczyk ${ }^{1}$ and Jacek Sein- Anand ${ }^{2}$ \\ ${ }^{1}$ Department of Biochemistry and Clinical Physiology, Medical University of Gdańsk, Poland \\ ${ }^{2}$ Department of Clinical Toxicology, Medical University of Gdańsk, Poland
}

*Corresponding author: Iwona Rybakowska, Department of Biochemistry and Clinical Physiology, Faculty of Health Sciences with Institute of Maritime and Tropical Medicine, Medical University of Gdańsk, Poland.

To Cite This Article: Iwona Rybakowska, Bartłomiej Łukasz, Magdalena Gregorczyk, Psychoactive Substances and Drugs-Medicament and Narcotic. Am J Biomed Sci \& Res. 2021 - 12(1). AJBSR.MS.ID.001703. DOI: 10.34297/AJBSR.2021.12.001703.

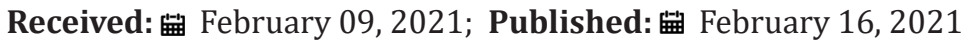

\begin{abstract}
Toxicological analysis is a complex set of tasks aiming to estimate how much time has elapsed since a drug was last consumed or to inform about its chronic consumption. It is also worth paying attention to the fact of using psychoactive substances induce euphoria or increase physical energy. The use of opioids to alleviate pain is complicated by the risk of severe adverse events and the large variability in dose requirements. Pharmacogenetics could be used to adapt pain medication based on an individual's genetic background. Many potential genetic markers related to xenobiotics metabolism have been described. Such predictors are especially of value for neonates and young children, in which case the assessment of efficacy is complicated by imprecise/poor communication. The knowledge of opioids metabolism is essential also for older patients and those with comorbidities, who receive multiple medications and may have impaired renal and hepatic function.
\end{abstract}

Keywords: Opioids, Pain, Cytochrome P450 family, Intoxication timeframe

\section{Introduction}

According to UNODC (United Nations Office on Drugs and Crime, 2017) World Drug Report 2017 in 20150.27 to $0.49 \%$ of global adults population (13-24 million people) had opiates use history, while for opioids were 0.59 to $0.88 \%$ (between 28 and 43 millions). Recent few years also show drastic effects of overly liberal distribution (from legal and illegal sources) of pharmaceutical opioids in US. So called opioid crisis resulted in an epidemic of overdose deaths and become "national public health emergency", as declared even politicians [1-3].

Interpretation of toxicological analysis allows to assess the period of substance ingestion and the time since opioids were used last by addicted individual. In relation to forensic medicine tasks important is to confirm if a scrutinized person is affected by given dug or not. Correct assessment of the intoxicant, and its metabolites, excretion time is crucial in this context. Products of heroin metabolism, including 6-acetylmorphine (6AM), morphine (MOR), morphine-6-glucuronide (M6G) and morphine-3-glucuronide (M3G), which appear in blood are examples [4]. On the other hand, opioids play important role in acute and chronic pain management [5]. Pharmacogenetic markers in this instance help to tailor clinical use of opioids, including Codeine, Tramadol, Tapentadol, Morphine, Oxycodone, Hydrocodone, Buprenorphine, Methadone [6].

\section{Heroin use}

Clinical and forensic toxicologists are confronted with establishing heroin use on a daily basis. This intoxicant and its metabolite, 6-acetylmorphine, have very short life-time in the organism. In addition, presence of morphine is not indicative towards any specific opiate, as heroine, codeine and morphine are metabolized to the same set of substances, namely MOR, M3G and M6G (Figure 1) [7]. Alternative approach to reveal heroine ingestion is a detection of other alkaloids contaminating illegally produced drug doses. Example of such impurity is acetylcodeine 
[8]. Similarly, presence of poppy opium ingredients, e.g. codeine, noscapine, papaverine or their metabolites in the suspect-derived samples suggest heroin use.

\section{Specific opioids used in acute pain treatment}

Morphine is an alkaloid derived from opium or breadseed poppy, Papaver somniferum and the most broadly used analgesic in clinical practice. This phenanthrene derivative is a weak base (pKa 7,9 ) which dissolves well in acidic aqueous environments but poorly in lipids, at physiological $\mathrm{pH}$. In humans $\mu$-opioid receptor binding affinity constant for morphine is $14 \mathrm{nM}$. It has a higher affinity to $\mu 1$ receptors, mediating anesthetic effects, than to $\mu 2$ type, linked to respiratory depression and negative digestive tract symptoms [9]. Morphine is absorbed in the intestines with a help of ATP-binding transporters in small intestine epithelial cells. Following absorption alkaloid molecules are captured by the liver and metabolized to M3G (40\%) and M6G (10\%) removed later with urine. The ration between morphine and its metabolites is dependent on the delivery route. Patients receiving oral doses show higher concentration of morphine glucuronides in plasma, comparing to parenteral administration. Liver dysfunction may lead to enhanced morphine bioavailability and, in consequence, shift the balance toward its metabolites. M6G is potentially more effective and longer-lasting MOR agonist, if compared to morphine, while M3G does not bind to opioid receptors. Former product of liver morphine transformations accumulates in blood and is able to penetrate blood-brain barrier, most probably harnessing active transport mechanisms. There it binds to opioid receptors with high affinity exerting strong anesthetic effects. Glycoprotein transporter is one of the suspects linked to regulating morphine and its metabolites concentrations in the brain [10]. If only morphine is administered, decrease of M6G plasma levels is slower than expected solely from its final half-life assessment. This observation suggest continuous creation of M6G from circulating drug. Total morphine clearance is relatively high and ranges from 75 to $118 \mathrm{~L} / \mathrm{h}$, in relation to $100 \mathrm{~L} / \mathrm{h}$ liver flow rate, for a $70 \mathrm{~kg}$ person [9]. Morphine can produce elevated clinical response in patients suffering from nephrological disorders, leading to acute toxic effects [11]. Age-dependent changes in kidney functioning may correspondingly alter morphine, metabolites ratio. Creatinine clearance in elderly rats receiving morphine or M6G was significantly reduced, in comparison with young animals. It suggests that lower medication dose may be required in older individuals to achieve comparable effects.

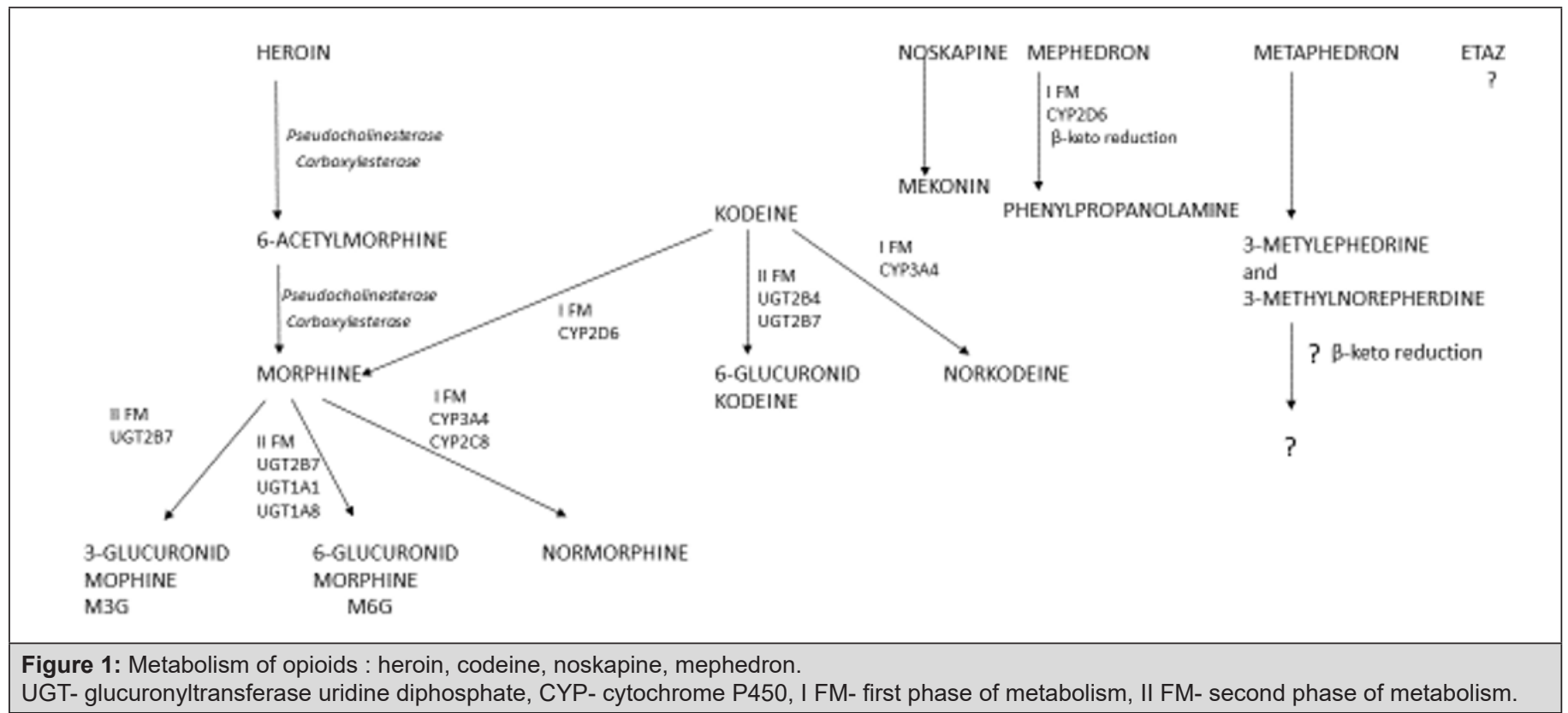

Tramadol is another analgesic acting through at least two mechanisms, namely as a weak opioids receptors (mainly $\mu$, but also $\delta$ and $\kappa$ ) agonist and a serotonin norepinephrine reuptake inhibitor (SNRI). Similar to codeine, it requires metabolic 'processing' to achieve full activity, via active metabolites. $85 \%$ of tramadol is metabolized in the liver by CYP3A4 and CYP2D6 to O-desmethyltramadol (O-DSMT). Majority (90\%) of remaining/ initial tramadol dose is removed by kidneys. The drug and its main metabolite exert anesthetic effects mainly through 'opioid' mechanism (mediated primarily by 0-DSMT) and by blocking serotonin and norepinephrine reuptake. Slow metabolizers can exhibit even 14-fold lower active metabolites concentration. Mutations in catechol-0-methyltransferase gene, product of which modulates dopaminergic, adrenergic and noradrenergic synaptic transmission, may interfere with drug activity.

\section{Mephedrone (4MMC)}

Mephedrone or 4-methylmethcathinone (4-MMC), or colloquially "mefa" was recognized in 2009 as the fourth most 
popular street drug in the UK (after marijuana, cocaine and ecstasy). At a similar time, it also appeared in Poland as an afterburner, adopted mainly by young people, regulars of parties and discos in 2010, however, he was banned. Mephedrone has a psychoactive effect and can cause serious side effects, especially when taken frequently, in large quantities and combined with alcohol and other stimulants. Mephedrone is an organic chemical compound (cathinone derivative), more specifically 2- (methylamino) -1(4-methylphenyl) propan-1-one with the sum formula C11H1SNO. Cathinone is an alkaloid found naturally in an African plant called cuvillaria (Catha edulis), famous for its stimulating properties. Mephedrone can be in the form of hydrochloride or sulfate, crystalline powder, capsules and pills. There are various routes of administration: nasal, oral, by injection, and rectal.

Mephedrone is a chemical compound used as a stimulant, i.e. a psychoactive substance with a stimulant effect. It belongs to the group of empatogens that cause characteristic emotional and social effects similar to those caused by ecstasy (MDMA). It belongs to the class of drugs such as amphetamine and less known cathinone. It was introduced to the market by the Israeli company Neorganics, which deals with the production of afterburners. It quickly spread in Europe as a "recreational" drug, but more countries began introducing legislation that considered it illegal.

Modified mephedrone is also available on the market of afterburners, the popularity of which has increased after the legalization of the original. Mephedrone is a drug people use to experience specific sensations. How does mephedrone work? The effects of its use include: increased alertness, nervousness, euphoria, excitement, talkativeness, openness, agitation, stronger sex drive, self-confidence. Its users compare the effects of mephedrone to something between cocaine and ecstasy. It works intensively, but quite briefly (for about an hour) - it makes the person who has taken it feel the need to reach for another dose.

Like other strong stimulants, this compound also contributes to side effects. The effects of mephedrone include increased sweating (even the term 'mephedrone sweat' works - it has a peculiar, sharp smell), headaches, heart palpitations, increased blood pressure, nausea, blue and cold fingers (symptom caused by peripheral vasospasm), muscle stiffness memory and concentration. People taking mephedrone may experience severe panic attacks, hallucinations and paranoia. The threat of these symptoms increases more as a result of sleep disorders (mephedrone as a stimulant causes insomnia), especially after party "marathons", which include taking the measure. An overdose of mephedrone causes psychiatric complications, including depressive syndromes, delusional psychoses, hallucinoses, aggressive behavior, hallucinations, anxiety, insomnia, anhedonia (inability to feel pleasure). Metabolism products of mephedrone may have neurotoxic and cardiotoxic effects. Several cases of mephedrone poisoning have already been registered. According to specialists, this is a relationship with a strong potential for psychological addiction, but it is still too poorly understood and there is a lack of research on this topic $[12,13]$.

\section{Metaphedrone}

3-Methylmethcathinone (metaphedrone, 3MMC) was first encountered in 2012, in Sweden [14] and is isomer of mephedrone. This synthetic cathinone has also similar psychostimulant properties to amphetamines, including euphoria, increased physical energy, alertness, feelings of empathy. Users prefer metaphedrone than mephedrone because of its strong effects.

\section{Etazen}

Etazen - an organic chemical compound from the opioid group is an analog of etonitazene. In studies on mice, it shows about 70 times stronger analgesic effect than morphine [15]. In June 2020, it was reported to the European Monitoring Center for Drugs and Drug Addiction after the use of this drug as a psychoactive substance was disclosed in Finland and Poland in March of the same year (Early Warning System Situation Report). It may cause death by inhibiting breathing.

\section{Conclusion}

Opioids are a group of natural or synthetic substances that bind to opioid receptors. We apply different opioids include codeine, morphine and heroin. The first two are opiates, the third is a product of chemical modification of morphine. Some opioids occur naturally in the human body (such as endorphins, enkephalins). Morphine is widely used in medicine as a remedy to combat moderate to severe pain, dyspnoea and cough. It is used, among others, to treat pulmonary edema, pain in a heart attack, and chronic pain. Due to its beneficial properties for pulmonary circulation, it is used to alleviate dyspnea in severe lung diseases and can be beneficial in the treatment of circulatory failure. Used outside of the treatment of ailments, it can lead to severe addiction, which is a potentially fatal disease. Another opioid codeine is a weak opioid used to treat mild to moderate pain and to relieve excessive coughing. We cannot forget about the new psychoactive substances like mephedrone, etazen used for better being, for getting a state of excitement. Another one is metaphedrone being synthetic cathinone which induce oxidative stress, GSH depletion, increased oxidized glutathion and caspase-3, $-8,-9$ activation which begins apoptotic cascade. Toxic effects of used opioid drugs or illegal opiates may appear. These problems renders further studies of opioids biotransformation and metabolism. 


\section{References}

1. Rummans TA, Burton MC, Dawson NL (2018) How Good Intentions Contributed to Bad Outcomes: The Opioid Crisis. Mayo Clinic Proceedings 93(3): 344-350.

2. Goodwin AJ (2018) Critical Care Outcomes Among Opioid Users: Hidden Sequelae.

3. Koenig KL (2018) The Opioid Crisis in America: Too much, too little, too late. West J Emerg Med 19: 557-558.

4. Dubois N, Hallet C, Seidel L, Demaret I, Luppens D, et al. (2015) Estimation of the Time Interval between the Administration of Heroin and Sampling of Blood in Chronic Inhalers. J Anal Toxicol 39(4): 300-305.

5. Madadi P, Koren G, Cairns J, Chitayat D, Gaedigk, A, et al. (2007) Safety of codeine during breastfeeding. Fatal morphine poisoning in the breastfed neonate of a mother prescribed codeine.

6. Mercadante S (2015) Opioid metabolism and clinical aspects. European Journal of Pharmacology 769: 71-78.

7. Stefanidou M, Athanaselis S, Spiliopoulou C, Dona A, Maravelias C (2010) Biomarkers of opiate use. Int J Clin Pract 64(12): 1712-1718.

8. O'Neal CL, Poklis A, Lichtman AH (2001) Acetylcodeine, an impurity of illicitly manufactured heroin, elicits convulsions, antinociception, and locomotor stimulation in mice. Drug Alcohol Depend 65(1): 37-43.
9. Sverrisdottir E, Lund TM, Olesen AE, Drewes AM, Christrup LL, Kreilgaard M (2015) A review of morphine and morphine-6-glucuronide's pharmacokinetic- pharmacodynamics relationship in experimental and clinical pain. Eur J Pharm 74: 45-62.

10. Fujita K, Ando Y, Yamamoto W, Miya T, Endo H, et al. (2010) Association of UGTB7 and ABCB1 genotypes with morphine-induced adverse effect drug reactions in Japanese patients with cancer. Cancer Chemother Pharmacol 65: 151-158.

11. King S, Forber K, Hanks GW, Chambers EJ (2011) A systematic review of the use of opiod medication for those with moderate to severe cancer pain and renal impairment: a European Palliative Care Research Collaborative opioid guidelines project. Palliat Med 25: 525-535.

12. Nordqvist Christian (2014) What is mephedrone? What are the effects of mephedrone?, Medical News Today, 9/09/2014.

13. Skowronek R. , Celinski R. , Chowaniec C., Skowronek A. (2012) Mephedrone and its new derivatives - toxicological, medical and legal aspects, Problems of Forensics, I 275 (1)

14. Backberg M, Lindeman E, Beck O, Helander A (2015) Characteristics of analytically confirmed 3-MMC-related intoxications from the Swedish STRIDA project. Clin Toxicol 53(1): 46-53.

15. Hunger A, Kebrle J, Rossi A, Hoffmann K (1960) Benzimidazol-Derivate und verwandte Heterocyclen. II. Synthese von 1-Aminoalkyl-2-benzylbenzimidazolen. Helvetica Chimica Acta 43(3): 800-809. 